\title{
Inibidores de síntese de giberelinas e crescimento de mudas de mangueira 'Tommy Atkins'
}

\author{
Synthesis inhibitors of gibberellins and mango 'Tommy Atkins' seedlings growth
}

\author{
Maria Aparecida do Carmo Mouco ${ }^{\mathrm{I}}$ Elizabeth Orika Ono ${ }^{\mathrm{II}}$ João Domingos Rodrigues ${ }^{\mathrm{II}}$
}

\section{RESUMO}

A mangicultura no semiárido brasileiro destacase pelos altos rendimentos, pela qualidade do fruto produzido e pela possibilidade de produção durante todo o ano, devido a condições climáticas e tecnologias para o manejo do crescimento vegetativo e da floração, com uso da irrigação, podas e retardantes vegetais. O paclobutrazol aplicado ao solo é utilizado no manejo da produção da mangueira na maioria dos pomares. Entretanto, há a necessidade de identificar retardantes vegetais que possam ser aplicados via foliar, de forma a minimizar os resíduos no solo e evitar o uso de quantidades inadequadas ao longo dos anos. Assim, este trabalho teve como objetivo avaliar retardantes vegetais aplicados via foliar na inibição do crescimento vegetativo de mudas de mangueira 'Tommy Atkins'. Prohexadione-Ca, etiltrinexapac e cloreto de chlormequat, aplicados via foliar, na dose de $1 \mathrm{~g}$ i.a. planta $^{-1}$, regulam o crescimento de ramos vegetativos, mas apresentam tempo diferenciado de atividade nas mudas, sendo 20 dias para prohexadione-Ca, 30 dias para cloreto de chlormequat e 45 dias para etil-trinexapac, nas condições em que o experimento foi conduzido.

Palavras-chave: Mangifera indica L, paclobutrazol, prohexadione-Ca, cloreto de chlormequat, etil-trinexapac.

\section{ABSTRACT}

The mango in the Brazilian semi-arid region stands out in the national scenario due to high yields and fruit quality, as well as to the possibility of all-year production due to climatic conditions and technologies which allow the management of plant growth and flowering through irrigation, pruning and use of plant growth regulators. Paclobutrazol applied to the soil is used for the production management of mangoes in most of the orchards. However, it is necessary to identify plant growth regulators which might be applied to the leaves, so as to minimize the risk of residues in the soil and to avoid inadequate application levels over several years. The present paper aimed at evaluating the effect of plant growth regulators applied to the leaves, regarding their efficiency for flowering management of mango 'Tommy Atkins'. Prohexadione-Ca, trinexapac-ethyl and chlormequat chloride at the dosage of $1.0 \mathrm{~g}$ a.i. plant ${ }^{-1}$ acts on the regulation of vegetative growth; the period of efficiency of the products applied to the leaves lasted 20 days for prohexadione-Ca, 30 days for chlormequat cloride, and 45 days for trinexapacethyl.

Key words: Mangifera indica L., paclobutrazol, prohexadioneCa, chlormequat chloride, trinexapac-ethyl.

\section{INTRODUÇÃO}

Na regulação da floração da mangueira e de outras culturas frutíferas decíduas, as giberelinas (GA) parecem ser os hormônios mais ativos, e altos níveis de GA inibem a floração e estimulam o crescimento vegetativo e o seu declínio promove a floração (DAVENPORT \& NUÑEZ-ELISEA, 1997). A maioria dos retardantes vegetais inibem a síntese de GA e podem, então, ser utilizados na redução do alongamento dos ramos e no manejo do crescimento vegetativo. Três tipos diferentes de retardantes vegetais que interferem na síntese da GA podem ser relacionados: primeiro, os compostos quaternários, como o cloreto de mepiquat e o cloreto de chlormequat (CCC), que inibem a conversão de geranil geranil difosfato para caureno; segundo, os compostos cíclicos contendo um

'Empresa Brasileira de Pesquisa Agropecuária, Embrapa Semiárido, 56302-970, Petrolina, PE, Brasil. E-mail: maria@cpatsa.embrapa.br. Autor para correspondência.

"Departamento de Botânica, Instituto de Biociências, Universidade Estadual Paulista (UNESP), Botucatu, SP, Brasil. 
nitrogênio, como o paclobutrazol (PBZ) e uniconazole, que inibem a passagem do caureno a $\mathrm{GA}_{12}$-aldeído; terceiro, os acilciclohexanodionas como o etiltrinexapac (TrixE) e o prohexadione-Ca (ProCa), que podem bloquear as reações finais do metabolismo de GA(RADEMACHER, 2004).

O PBZ é o retardante vegetal utilizado no manejo da produção da mangueira na maioria dos pomares sob as condições semiáridas do Nordeste, permitindo o escalonamento da produção de manga. Apresenta como inconvenientes a necessidade de aplicação no solo, já que é ineficiente quando aplicado via foliar (MOUCO \& ALBUQUERQUE, 2005), e a maior persistência na planta e no solo (RADEMACHER et al., 2006).

O ProCa (cálcio 3-óxido-4-propionil-5-oxo3-ciclohexene carboxilato) interfere no processo de alongamento da célula (EVANS et al., 1999) e tem tempo de permanência em macieiras, sob condições climáticas típicas, entre 10 e 14 dias. A translocação de forma acrópeta no xilema traz como benefício o controle efetivo do crescimento vegetativo em distintas partes da planta como resultado das pulverizações e, devido a sua baixa persistência e ao tipo de movimento dentro das plantas, pouco ou nenhum resíduo é encontrado nos frutos. Diferente do PBZ e de outros compostos mais estáveis, a vida média do ProCa em solo com atividade microbiana é menor que 24 horas (RADEMACHER et al., 2006). O ProCa foi eficiente em reduzir o tamanho dos entrenós em macieiras e pereiras (BASAK, 2004; COSTA et al., 2004), e o efeito foi mais rápido que nas aplicações com CCC e PBZ (ASIN \& VILARDELL, 2006; DECKERS et al., 2005).

O TrixE (4-ciclopropil hidroxi metileno ácido 3,5-dioxi ciclo hexano carboxílico) é um retardante vegetal que atua no final do processo de síntese de GA. Foi testado em macieiras por MAXSON \& JONES (2002), que observaram redução no crescimento vegetativo de ramos depois de 10 a 14 dias da pulverização, sendo a inibição mantida por mais 30 dias. Os autores concluíram que concentrações mais altas, na faixa de $1 \mathrm{~g} \mathrm{~L}^{-1}$, são as mais eficientes.

A eficiência do CCC (cloreto de 2-cloroetil trimetil-amônio) no manejo da floração da mangueira, relatada por DAVENPORT \& NUÑEZ-ELISEA(1997), não apresentou consistência ao longo dos anos. No entanto, experimento conduzido em abacateiro por PENTER \& STASSEN (1999) mostrou que a aplicação de retardantes vegetais apresentou efeito na inibição da brotação vegetativa e no manejo da floração; nesses resultados, o CCC foi mais eficiente no incremento do rendimento e tamanho de fruto que o paclobutrazol e uniconazole. THERON et al. (1998), trabalhando com a cultura da pereira e testando pulverizações de CCC em concentrações que variaram de 1 a $4 \mathrm{~g} \mathrm{~L}^{-1}$, concluíram que este é eficiente em regular o crescimento vegetativo, promover a floração e aumentar o estabelecimento de frutos.

Pelo exposto, objetivou-se, com o presente trabalho, avaliar o efeito de diferentes retardantes vegetais aplicados via foliar na inibição do crescimento vegetativo em mudas de mangueira 'Tommy Atkins', na região semiárida do Nordeste brasileiro.

\section{MATERIAL E MÉTODOS}

O experimento foi conduzido entre outubro de 2005 e julho de 2006, em casa-de-vegetação da Embrapa Semiárido, em Petrolina, Pernambuco (PE). As condições de temperatura e umidade do ar foram monitoradas por um termo-higrógrafo; os dados indicaram médias de temperatura máxima e mínima de $35 \pm 3^{\circ} \mathrm{C}$ (dia) e $22 \pm 2^{\circ} \mathrm{C}$ (noite), e umidade relativa entre $85 \pm 5 \%$ e $45 \pm 5 \%$.

O substrato utilizado nos vasos foi preparado com mistura de solo, esterco de curral e areia na proporção de 1:1:1, sendo as mudas transplantadas para os vasos quando apresentaram um fluxo vegetativo após a realização da enxertia de garfos da cultivar 'Tommy Atkins' sobre a variedade Espada. Antes da aplicação dos tratamentos, foram realizadas duas pulverizações com nitrato de potássio a 2,5\% (25/ 10/2005 e 04/11/2005) para induzir brotação vegetativa uniforme. O experimento foi implantado depois da emissão e expansão do segundo fluxo vegetativo das mudas, ocorrido em 28/11/2005, com a aplicação dos retardantes vegetais, na dose de $1,0 \mathrm{~g}$ i.a. planta $^{-1}$, em uma única ou em duas aplicações de $0,5 \mathrm{~g}$ i.a. planta $^{-1}$, intercaladas de 30 dias. O delineamento foi inteiramente casualizado, com sete tratamentos e cinco repetições, sendo a unidade experimental representada por uma planta. Os tratamentos testados são listados a seguir, sendo utilizada uma sigla para cada um dos retardantes, ProCa, TrixE e CCC, seguido do número de pulverizações.

T1- ProCa 2 (0,5g i.a. planta $^{-1}+0,5$ g i.a. planta $\left.^{-1}\right)$

T2- ProCa 1 (1,0g i.a. planta $\left.^{-1}\right)$

T3- TrixE 2 (0,5g i.a. planta ${ }^{-1}+0,5$ g i.a. planta $\left.^{-1}\right)$

T4- TrixE 1 (1,0g i.a. planta $\left.{ }^{-1}\right)$

T5- CCC 2 (0,5g i.a. planta ${ }^{-1}+0,5$ g i.a. planta $\left.^{-1}\right)$

T6- CCC 1 (1,0g i.a. planta $\left.{ }^{-1}\right)$

T7- Controle

A quantidade de água para a irrigação foi determinada pela pesagem do solo (sem o vaso) e pelo cálculo de volume de água para deixá-lo com $80 \%$ da capacidade de campo; a irrigação foi feita por 
gotejamento, e a necessidade diária foi estimada também por meio da pesagem dos vasos com o solo e a planta (amostragem). As avaliações envolveram as anotações do número e comprimento dos fluxos emitidos depois do início das aplicações dos retardantes vegetais, além do número de folhas, da área foliar, dos sintomas de fitotoxicidade e da massa fresca de folhas, dos ramos e das raízes após 190 dias. Os dados obtidos nas avaliações foram submetidos à análise de variância; para o teste de médias, foi utilizado o Tukey, a 5\% de probabilidade de erro.

\section{RESULTADOS E DISCUSSÃO}

Na tabela 1, observa-se que os tratamentos que envolveram duas pulverizações de CCC (T5) e do TrixE (T3 e T4) reduziram o comprimento do ramo principal em até 34 e $41 \%$ em relação ao controle. As pulverizações com TrixE foram as mais eficientes em inibir a emissão de novos fluxos vegetativos, reduzindo também o número de folhas e a área foliar das mudas de mangueira 'Tommy Atkins'. MAXSON \& JONES (2002) também encontraram reduções no crescimento de ramos de macieira pulverizadas com TrixE, sendo a inibição mantida por mais de 30 dias. Contrariamente, o CCC não impediu a emissão de novos fluxos vegetativos da mangueira, provocando efeito só no encurtamento dos entrenós (Tabela 1).

Com relação ainda ao número de fluxos emitidos durante o período do experimento, observase, na tabela 1 , que as mudas tratadas com TrixE emitiram, em média 2,4 fluxos vegetativos, e aquelas que receberam o ProCa e CCC emitiram 4,1 e 4,2 fluxos, respectivamente. Essas diferenças podem ser atribuídas ao tempo de atividade de cada um dos retardantes testados dentro da planta, já que as avaliações de brotação realizadas durante o experimento mostraram que, aos 30 dias após a primeira pulverização, nos tratamentos com ProCa, as plantas já tinham emitido uma segunda brotação vegetativa, com comprimento médio de $10 \mathrm{~cm}$. Nesse momento, as plantas pulverizadas com CCC apresentavam somente início de novas brotações, e as tratadas com o TrixE mantinham as gemas dos ramos em repouso, enquanto as plantas do controle já apresentavam novo fluxo vegetativo totalmente expandido. Em 28/12/2005, também foi feita a segunda pulverização nas plantas dos tratamentos ProCa 2, TrixE 2 e CCC 2, na dose de 0,5 g i.a. planta $^{-1}$.

Aos 60 dias da primeira e trinta da segunda pulverização, as plantas do T1 (ProCa 2) somente haviam iniciado a emissão de nova brotação vegetativa, mas as plantas do tratamento ProCa 1 (1g i.a. planta ${ }^{-1}$ aplicado numa única pulverização no início do experimento, 60 dias antes) já apresentavam fluxos expandidos. Nota-se que, independentemente da concentração, o efeito em inibir a brotação vegetativa da mangueira com o ProCa teve duração de 20 a 25 dias. O tratamento T4 (TrixE 1), que recebeu o TrixE em uma única aplicação de $1,0 \mathrm{~g}$ i.a. planta ${ }^{-1}$, apresentou brotações vegetativas aos 45 dias da pulverização. Quando a dose de TrixE foi dividida e uma segunda pulverização de $0,5 \mathrm{~g}$ i.a. planta $^{-1}$ foi feita depois de 30 dias, as brotações vegetativas só foram observadas também após 45 dias, ou seja, 75 dias da primeira. Essas observações permitem inferir um período maior de atividade do TrixE em mudas de mangueira 'Tommy Atkins' e estão de acordo com os resultados do estudo de MAXSON \& JONES (2002) em macieiras. Já nas mudas tratadas com CCC, as primeiras brotações surgiram aos 30 dias da primeira pulverização, principalmente no tratamento que recebeu duas

Tabela 1 - Comprimento do ramo principal (cm), número total de folhas, área foliar $\left(\mathrm{cm}^{2}\right)$ e número de fluxos no ramo principal. Mangueira da cultivar ‘Tommy Atkins'. Petrolina, PE. 2005/2006.

\begin{tabular}{|c|c|c|c|c|}
\hline Tratamentos & Ramo principal (cm) & Número total de folhas & Área foliar $\left(\mathrm{cm}^{2}\right)$ & Fluxos no ramo principal \\
\hline 1- ProCa $2^{\mathrm{w}}$ & $108,6 a^{y}$ & $189,6 \mathrm{ab}$ & $10606,6 \mathrm{ab}$ & $4,4 \mathrm{a}$ \\
\hline 2- ProCa 1 & 99,2 a & 225,8 a & 11703,2 a & $3,8 \mathrm{ab}$ \\
\hline 3- TrixE 2 & $61,2 \mathrm{~b}$ & $82,2 \mathrm{~cd}$ & $5394,9 \mathrm{~cd}$ & $2,2 \mathrm{c}$ \\
\hline 4- TrixE 1 & $61,6 \mathrm{~b}$ & $72,8 \mathrm{~d}$ & $4632,9 \mathrm{~d}$ & $2,6 \mathrm{bc}$ \\
\hline 5- CCC 2 & $68,8 \mathrm{~b}$ & $103,6 \mathrm{~cd}$ & 7923,3 bcd & 4,2 a \\
\hline 6- CCC 1 & $84,6 \mathrm{ab}$ & 131,6 bcd & $8743,3 \mathrm{abc}$ & 4,2 a \\
\hline 7- Controle & 104,6 a & 145,4 bc & $9138,1 \mathrm{ab}$ & $4,0 \mathrm{a}$ \\
\hline CV (\%) & 15,1 & 25,1 & 19,9 & 16,5 \\
\hline
\end{tabular}

${ }^{\text {w }}$ Os tratamentos T1, T3 e T5 (ProCa 2, TrixE 2 e CCC 2) receberam o retardante vegetal em duas pulverizações, com 0,5g i.a. cada. Os tratamentos T2, T4 e T6 (ProCa 1, TrixE 1 e CCC 1) receberam a dose de 1g i.a. de uma única vez.

${ }^{y}$ Média de cinco repetições, seguidas da mesma letra, não diferem estatisticamente entre si (Tukey P=0,05). 
aplicações de 0,5 g i.a. planta ${ }^{-1}$. No tratamento em que foi feita uma única pulverização de 1,0 g i.a. planta $^{-1}$ (CCC 1), as mudas mostraram-se mais vigorosas, sendo observadas também mais brotações laterais.

Os tratamentos T2 (ProCa 1) e T6 (CCC 1), que receberam $1,0 \mathrm{~g}$ i.a. planta $^{-1}$ em única pulverização, apresentaram maior número de folhas e área foliar do que os tratamentos que receberam a mesma dose aplicada em duas pulverizações, T1 (ProCa 2) e T5 (CCC 2), sem diferirem estatisticamente (Tabela 1). Nesse caso, provavelmente, a reaplicação manteve os níveis de GAs ativas menores, inibindo a emissão de novas folhas, como também doses maiores podem comprometer a eficiência do ProCa e CCC. Doses mais baixas e a reaplicação do ProCa são mais eficientes no controle do comprimento dos ramos e da área foliar em macieiras (GREENE, 2008; ZADRAVEC et al., 2008). Os tratamentos ProCa 1 e 2 apresentaram número de folhas superior ao controle, mas significativamente apenas nas plantas que foram pulverizadas uma única vez (ProCa 1) (Tabela 1).

O TrixE, quando aplicado (1,0g i.a. planta $\left.^{-1}\right)$ de uma única vez (TrixE 1), reduziu o número de folhas e a área foliar das plantas quando comparado com o T3 (TrixE 2), em que foram feitas duas pulverizações de 0,5g i.a. planta ${ }^{-1}$, com intervalo de 30 dias. O TrixE em uma (TrixE 1) e em duas (TrixE 2) pulverizações reduziu o número de folhas das mudas em 49 e 44\% em relação ao controle, e apenas o tratamento com TrixE numa única aplicação induziu uma redução significativa em relação ao controle. Os tratamentos TrixE 1 e 2 reduziram significativamente a área foliar em relação ao controle, em 41 e 49\%, respectivamente. Esse resultado deve-se possivelmente ao efeito de inibir a emissão de novas folhas. O modo de ação do TrixE envolve acúmulo de moléculas do ingrediente ativo na região do meristema intercalar, com subsequente inibição da biossíntese de giberelina e menor expansão da célula nas regiões da base e na bainha de folhas (KAUFMANN, 1986).

Os tratamentos T5 e T6 (CCC 2 e 1) reduziram o número de folhas, mas essa diferença não foi significativa, correspondendo a 29 e 9,5\%, respectivamente, dos valores obtidos com as plantas do controle, em que a aplicação em dois momentos, com intervalo de 30 dias, foi a mais eficiente em regular o crescimento (Tabela 1).

A afirmação de que os retardantes vegetais, inibidores de síntese de giberelinas, quando aplicados em altas doses, podem reduzir o número de folhas formadas foi confirmada por REEKIE et al. (2005), trabalhando com o ProCa em plantas de morango, como também por DEYTON et al. (1991), os quais mostraram que aplicações de PBZ, também em plantas de morango, na dose de $1,2 \mathrm{~g} \mathrm{~L}^{-1}$, reduziram o número de folhas com maior intensidade do que em pulverizações entre $75 \mathrm{e}$ $600 \mathrm{mg} \mathrm{L}^{-1}$.

Aos 90 dias da primeira pulverização com os retardantes vegetais e 60 dias da segunda e última, notáveis diferenças no crescimento das plantas entre os tratamentos com relação ao controle foram observadas (Figura 1), confirmando os resultados apresentados na tabela 1 . As mudas só foram colhidas 100 dias depois, ou seja, 190 dias do início do experimento e durante esse período novas brotações surgiram nos tratamentos que receberam ProCa e CCC. Entretanto, as mudas tratadas com TrixE não voltaram a emitir novas brotações vegetativas, sendo observados danos de queima nas folhas, mas que estiveram associados principalmente à presença de folhas imaturas no momento da pulverização.

Os tratamentos com o CCC e TrixE, independentemente se aplicados em uma ou em duas pulverizações, reduziram significativamente a massa fresca total das plantas, em média, para 70 e $44 \%$ dos valores obtidos com o controle, respectivamente (Figura 2). A redução induzida pelo TrixE na massa fresca das plantas foi maior do que a promovida pelos demais retardantes. O resultado na massa fresca total das plantas, em todos os tratamentos, quando comparado com o controle, foi devido, tanto ao efeito dos retardantes vegetais na brotação da parte aérea, quanto no crescimento das raízes. Quando se avalia o efeito dos retardantes nos diferentes componentes da parte aérea, folhas e ramos, nota-se que as diferenças observadas entre CCC e TrixE se devem apenas à massa de folhas (Figura 2).

O ProCa também reduziu a altura de planta e área foliar na cultura do morango, mas as diferenças entre as plantas tratadas e o controle, gradualmente, decresceram após 28 dias. Entretanto, aos 42 dias, as plantas tratadas ainda estavam 15 a $30 \%$ menores que o controle, sem diferirem significativamente, e a massa das raízes das plantas tratadas era significativamente maior que a massa das plantas do controle. Como resultado, a massa das plantas no final do experimento era igual, independentemente do tratamento. Com o desenvolvimento das plantas, houve maior alocação progressiva para as raízes e menor para a parte aérea, e a percentagem de alocação para os tecidos das raízes, ramos e folhas foi diferente entre as plantas tratadas e a do controle (REEKIE et al., 2005).

O ProCa reduziu em $50 \%$ a altura de macieira, sob condições de campo, mas não o número de folhas; a massa das plantas pode ser reduzida pelo ProCa, mas depende de quando são feitas as pulverizações (GUAK et al., 2001). O ProCa também decresceu a área foliar 

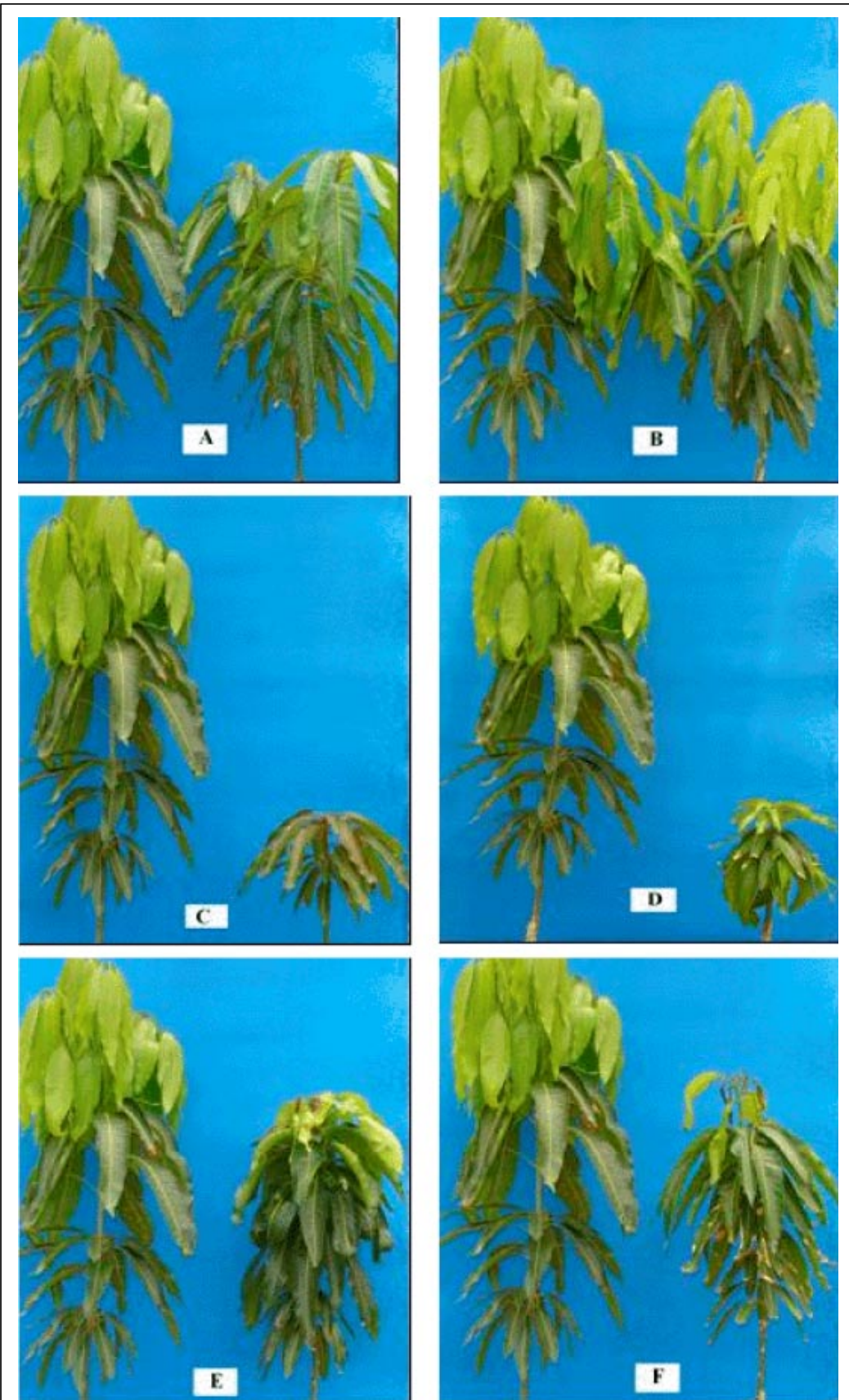

Figura 1 - Mudas de mangueira da cultivar 'Tommy Atkins’ submetidas aos tratamentos com retardantes vegetais e comparadas com o controle (planta da esquerda). $\mathrm{T} 1=\operatorname{ProCa} 2$ (A); T2= ProCa 1 (B); T3= TrixE 2 (C); T4= TrixE 1 (D); T5= CCC 2 (E); T6= CCC 1 (F). Embrapa Semiárido, Petrolina, PE. 2006.

específica, e as macieiras tratadas exibiram a mais alta taxa fotossintética e o incremento de $50 \%$ na absorção de $\mathrm{CO}_{2}$ (SABATINI et al., 2003).

Nas práticas visando ao escalonamento da produção da mangueira, ALBUQUERQUE et al. (2002) recomendam que, pelas condições climáticas encontradas no semiárido nordestino, é necessário que o manejo da indução floral seja iniciado em plantas com ramos maduros, no mínimo com 80 a 100 dias de idade, o que pode ser possível em mangueiras adultas, com uma única aplicação de PBZ no solo. No entanto, com base nos resultados obtidos, são necessárias quatro pulverizações do ProCa a cada 20 dias, ou três pulverizações do CCC com intervalo de 30 dias e, no 


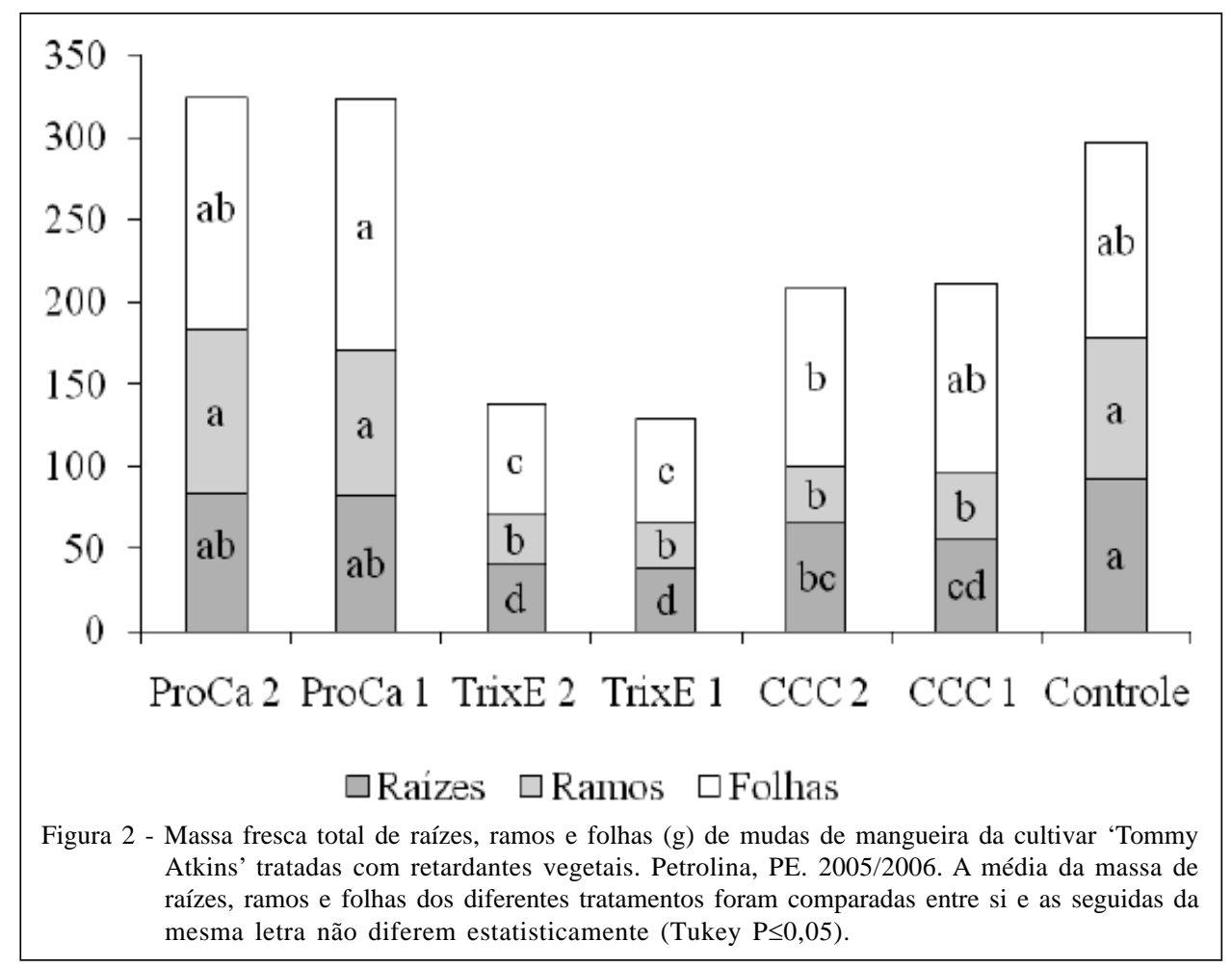

caso do TrixE, duas a cada 45 dias, para manter a muda de magueira sem emissão de novos fluxos vegetativos por 90 dias.

\section{CONCLUSÕES}

Prohexadione-Ca, cloreto de chlormequat e etil-trinexapac, aplicados via foliar, foram eficientes em regular o crescimento vegetativo de ramos em mudas de mangueira 'Tommy Atkins'.

\section{REFERÊNCIAS}

ALBUQUERQUE, J.A.S. et al. Indução floral. In: GENU, P.J. de C.; PINTO, C.A. de Q. (Ed.). A cultura da mangueira. Brasília, DF: Embrapa Informação Tecnológica, 2002. Cap.13, p.259-276.

ASIN, L.; VILARDELL, P. Effect of paclobutrazol and Prohexadione-Calcium on shoot growth rate and growth control in "Blanquilla" and "Conference" Pear. Acta Horticulturae, Leuven, n.727, p.133-138, 2006.

BASAK, A. Growth and fruting of "Elstar" apple trees in response to prohexadione calcium depending on the rootstock. Acta Horticulturae, Leuven, n.653, p.117-126, 2004.

COSTA, G.et al. Prohexadione-Ca controls vegetative growth and cropping performance in pear. Acta Horticulturae, Leuven, n.653, p.35-40, 2004.
DAVENPORT, T.L.; NUÑEZ-ELISEA, R. Reproductive phisiology. In: LITZ, R.E. The mango. Wallingford: CAB International, 1997. p.69-121.

DECKERS, T. et al. Natural or chemical growth regulation in pear. Acta Horticulturae, Leuven, n.671, p.503-516, 2005.

DEYTON, D.E. et al. Strawberry growth and photosynthetic responses to paclobutrazol. HortScience, Alexandria, v.26, p.1178-1180, 1991.

EVANS, L. et al. Mode of action, metabolism and uptake of BAS-125W, prohexadione-calcium. HortScience, Alexandria, v.34, n.7, p.1200-1201, 1999.

GREENE, D.W. The effect of repeat annual applications of Prohexadione-calcium on fruit set, return bloom, and fruit size of apples. HortScience, Alexandria, v.43, p.376-379, 2008.

GUAK, S. et al. Growth, allocation of $\mathrm{N}$ and carbohydrates, and stomatal conductance of greenhouse grown apple treated with prohexadione-Ca and gibberellins. Journal Horticultural Science Biotechnology, Ashford, v.76, p.746-752, 2001.

KAUFMANN, J.E. The role of PGR science in chemical vegetation control. Proceeding Plant Growth Regulation Society of American, St. Petersburg, v.13, p.2-14, 1986.

MAXSON, K.L.; JONES, A.L. Management of fire blight with gibberellin inhibitors and sar inducers. Acta Horticulturae, Leuven, n.590, p.217-223, 2002.

MOUCO, M.A.do C.; ALBUQUERQUE, J.A.S. de. Efeito do paclobutrazol em duas épocas de produção da mangueira. Bragantia, Campinas, v.64, n.2, p.219-225, 2005. Disponível em: <http:// www.scielo.br/scielo.php?script=sci_arttext\&pid=S0006- 
$87052005000200008 \& \operatorname{lng}=$ en\&nrm=iso $>$. Acesso em 30 set. 2009. doi: $10.1590 /$ S0006-87052005000200008.

PENTER, M.G.; STASSEN, P.J.C. Chemical manipulation as part of a management programme for improved fruit yield and quality in avocado orchards. South African Avocado Growers Association Yearbook, Pretoria, v.22, p.69-75, 1999. Disponível em: <http://www.avocadosource.com/Journals/ SAAGA/SAAGA_1999/SAAGA_1999_PG_069-075.pdf >. Acesso em 30 set.2009.

RADEMACHER, W. Chemical regulation of shoot growth in fruit trees. Acta Horticulturae, Leuven, n.653, p.29-32, 2004.

RADEMACHER, W. et al. Prohexadione-Ca: modes of action of a multifuncional plant bioregulator for fruit trees. Acta Horticulturae, Leuven, n.727, p.97-106, 2006.
REEKIE, J.Y. et al. Prohexadione-calcuium modifies growth and increases photosynthesis in strawberry nursery plants. Canadian Journal Plant Science, Ottawa, v.85, p.671-677, 2005.

SABATINI, E. et al. Prohexadione-Ca positively affects gas exchanges and chlorophyll content of apple and pear trees. European Journal Horticultural Science, Hannover, v.68, p.123-128, 2003.

THERON, K.Y. et al. Effect of autumm application of chlormequat on reproductive bud development, fruit set and production of the pear cv Dyenne du Comice. Acta Horticulturae, Leuven, n.475, p.251-264, 1998.

ZADRAVEC, P. et al. Vegetative growth,yield and fruit quality of 'Gala' apple treated with Regalis (prohexadione-Ca). Acta Horticulturae, Leuven, n.774, p.287-290, 2008. 